\title{
THE BETA-NEUTRINO CORRELATION IN SODIUM-21 AND OTHER NUCLEI
}

\author{
P.A. Vetter, J. Abo-Shaeer, S.J. Freedman, R. Maruyama \\ Nuclear Science Division, Lawrence Berkeley National Laboratory, \\ Berkeley, $C A 94618$ \\ *E-mail: pavetter@LBL.GOV \\ www.lbl.gov/nsd
}

\begin{abstract}
We have measured the $\beta-v$ correlation coefficient, $a_{\beta_{v}}$, in ${ }^{21} \mathrm{Na}$ using a laser-trapped sample. We measure the energy spectrum of the recoil nuclei by measuring their time-offlight in coincidence with the atomic electrons shaken off in beta decay. High detection efficiency of these low-energy electrons allows good counting statistics, even with low trap density, which suppresses the photoassociation of molecular sodium, which can cause a large systematic error. Our measurement, with a $1 \%$ fractional uncertainty, agrees with the Standard Model prediction but disagrees with our previous measurement which was susceptible to error introduced by molecular sodium. We summarize precise measurements of $a_{\beta v}$ and their consequences for searches for Beyond Standard Model scalar and tensor current couplings.
\end{abstract}

\section{Introduction}

Some Beyond Standard Model theories predict scalar and tensor couplings of leptons and quarks which could be detected in measurements of the beta-neutrino momentum correlation [1-3]. Short-lived isotopes in neutral atom traps are an appealing source for the study of $\beta$ decay correlations, and many experiments have been performed and proposed in such systems [4-6]. Ideally, recoiling daughter nuclei and $\beta$ particles emerge from the trap with no scattering and propagate in ultra-high vacuum. Decays occur essentially at rest and are localized in a small volume. The source location and spatial distribution can be monitored optically. The nuclear polarization state can be manipulated using optical pumping. The trapped atoms are isotopically pure, and very little background activity is present when the fiducial detection region is restricted to a zone around the trap (otherwise, atoms scattered out of the trap act as background activity with poorly characterized source distribution and polarization).

A previous measurement of the beta-neutrino correlation in laser-trapped ${ }^{21} \mathrm{Na}$ found a value $3.6 \sigma$ smaller than that calculated from the "V minus $\mathrm{A}$ " current 
coupling of the Standard Model [7]. The results suggested a dependence on the number of atoms: at lower trap populations, $a_{\beta v}$ was larger, and if extrapolated to zero trap population, the result agreed with the calculated value. Repeating the experiment using a more efficient detection system suggested in [8], we found convincing evidence that the previous measurement of $a_{\beta v}$ was distorted by events originating from cold, trapped molecules of ${ }^{21} \mathrm{Na}_{2}$.

\section{The Beta-Neutrino Correlation in the Electroweak Standard Model}

In a source with no net nuclear polarization or tensor alignment, the $\beta-v$ correlation can be inferred from the $\beta$ decay rate $[9,10]$

$$
\begin{array}{r}
\frac{d^{3} \Gamma}{d E_{e} d \Omega_{e} d \Omega_{v}} \propto F\left(Z, E_{e}\right) p_{e} E_{e}\left(E_{0}-E_{e}\right)^{2} \\
\times\left(f_{1}\left(E_{e}\right)+a_{\beta v}\left(E_{e}\right) \frac{\vec{p}_{e} \cdot \vec{p}_{v}}{E_{e} E_{v}}+b_{\text {Fierz }}\left(E_{e}\right) \frac{m_{e}}{E_{e}}\right) .
\end{array}
$$

Here $\left(E_{e}, \vec{p}_{e}\right)$ and $\left(E_{v}, \vec{p}_{v}\right)$ are the $\beta$ and $v$ 4-momentum, $E_{0}$ is the $\beta$ decay endpoint energy, $m_{e}$ is the electron mass, and $F\left(Z, E_{e}\right)$ is the Fermi function. In the allowed approximation, $f_{1}$, the $\beta-v$ correlation coefficient $\left(a_{\beta v}\right)$ and Fierz interference term $\left(b_{\text {Fierz }}\right)$ are independent of $E_{e}$. Their values are determined by the weak coupling constants $C_{i}$ and $C_{i}^{\prime}\{i=\operatorname{scalar}(S)$, vector $(V)$, tensor $(T)$, and axial-vector $(A)\}$, and by the Fermi (Gamow-Teller) nuclear matrix elements, $M_{F}$ $\left(M_{G T}\right)$ [9]. For a mixed Fermi/Gamow-Teller beta decay,

$$
\begin{gathered}
a_{\beta v}=\left(\left|M_{F}\right|^{2}\left(\left|C_{V}\right|^{2}+\left|C_{V}^{\prime}\right|^{2}-\left|C_{S}\right|^{2}-\left|C_{S}^{\prime}\right|^{2}\right)\right. \\
\left.-\frac{1}{3}\left|M_{G T}\right|^{2}\left(\left|C_{A}\right|^{2}+\left|C_{A}^{\prime}\right|^{2}-\left|C_{T}\right|^{2}-\left|C_{T}^{\prime}\right|^{2}\right)\right) \xi^{-1}
\end{gathered}
$$

where

$$
\begin{aligned}
& \xi=\left|M_{F}\right|^{2}\left(\left|C_{V}\right|^{2}+\left|C_{V}^{\prime}\right|^{2}+\left|C_{S}\right|^{2}+\left|C_{S}^{\prime}\right|^{2}\right) \\
& +\left|M_{G T}\right|^{2}\left(\left|C_{A}\right|^{2}+\left|C_{A}^{\prime}\right|^{2}+\left|C_{T}\right|^{2}+\left|C_{T}^{\prime}\right|^{2}\right),
\end{aligned}
$$

and

$$
\begin{array}{r}
b_{\text {Fierz }}= \pm 2 \sqrt{1-(Z \alpha)^{2}} \operatorname{Re}\left[\left|M_{F}\right|^{2}\left(C_{S} C_{V}^{*}+C_{S}^{\prime} C_{V}^{\prime *}\right)\right. \\
\left.+\left|M_{G T}\right|^{2}\left(C_{T} C_{A}^{*}+C_{T}^{\prime} C_{A}^{\prime *}\right)\right] \xi^{-1} .
\end{array}
$$


In the Standard Model (SM), $C_{V}$ and $C_{A}$ are almost purely real, ${ }^{\mathrm{a}} C_{V}=C_{V}^{\prime}=1$, and $C_{A}=C_{A}^{\prime} \approx-1.27$ (from experiments), and all other coupling constants are zero. Experimental limits on scalar and tensor couplings predicted by some SM extensions are model dependent, and not necessarily stringent [1]. If present, these couplings would alter $a_{\beta v}$ either through the quadratic dependence on $C_{S}$ and $C_{T}$ or through the helicity-sensitive Fierz interference terms. Searching for new treelevel lepton-quark interactions in nuclear beta decay is tough because there are many different couplings to measure: $C_{V}$ and $C_{A}$, the opposite chirality (primed) terms, and limits on $C_{S}$ and $C_{T}$. Absent a predicted symmetry group, we have 19 different couplings to measure. Naïvely, at tree-level, each coupling constant would be related to a new boson like $1 / M_{W^{\prime}}^{2}$, so that measurements with a fractional precision of $1 \%$ of $C_{V}$ and $C_{A}$ constrain physics at an energy scale ten times higher than the electroweak scale.

Interpreting measurements of beta-decay correlations to test the Standard Model demands high precision auxiliary nuclear data. Several corrections alter the allowed approximation prediction of $a_{\beta v}$ and give $E_{e}$ dependence to $f_{1}, a_{\beta v}$, and $b_{\text {Fierz }}$ at the $1 \%$ level. The input data include ground and excited state decay branching ratios, half-life, total decay energy, electron capture branching ratio, radiative corrections to order $\alpha$, isospin symmetry breaking corrections, and magnetic moments of parent and daughter to estimate the weak magnetism contribution. Measurements of decay correlations to better than $1 \%$ precision will be limited by the precision of these auxiliary inputs. We should view these nuclear beta decay systems as a significant frontier for testing Beyond Standard Model physics, and advocate for new, high precision input data as a significant use of a potential RIA/FRIB nuclear accelerator facility.

\section{3. $\beta-v$ measurement technique}

The experiment apparatus have been described in $[7,8,11]$. The measurement technique is at root a momentum spectrometer for the recoil nuclei. The magnetooptic trap (MOT) is located between two microchannel plates (MCPs) and several electrodes which form a focusing electric field in the region of the trap. The $\beta$ decay leaves ${ }^{21} \mathrm{Ne}$ in a variety of charge states through shakeoff and Auger processes [11]. The electric field accelerates the ionized recoil nuclei to one MCP and the low-energy electrons shaken off by the ${ }^{21} \mathrm{Ne}$ towards the second MCP. A trigger from the electron MCP starts a time to amplitude converter, with a stop signal

${ }^{\mathrm{a}} C_{V}$ and $C_{A}$ acquire a tiny imaginary part from the complex phase in the CKM matrix in heavy quark flavor mixing. For the rest of this discussion, we assume they are purely real, and the non-Standard Model couplings for which we derive limits are also real, i.e. time-reversal invariant. 
provided by the ${ }^{21} \mathrm{Ne}$ ions detected by the ion MCP. The $\beta-v$ correlation can be inferred from the time-of-flight (TOF) spectrum since aligned lepton momenta (caused by the $a_{\beta v}$ term in Eq. 1) result in larger nuclear recoil energies. A CCD camera acquires images of the MOT, and the trap population is inferred from the measured intensity of the fluorescence.

\section{Generating Fit Templates}

To interpret the time-of-flight spectra, we use Monte-Carlo simulations to generate template TOF curves for the two kinematic terms in Eq. 1 with $a_{\beta v}=0$ and $a_{\beta v}=0.553$. The recoil ion TOF data are fit to a linear combination of these two template spectra to determine $a_{\beta v}$. The Monte-Carlo generated TOF template spectra for beta decays to ${ }^{21} \mathrm{Ne}^{+}$, are shown in Fig. 1. Electron capture events are included with a $0.087 \%$ branching ratio. ${ }^{\mathrm{b}}$ Measuring the size and location of the

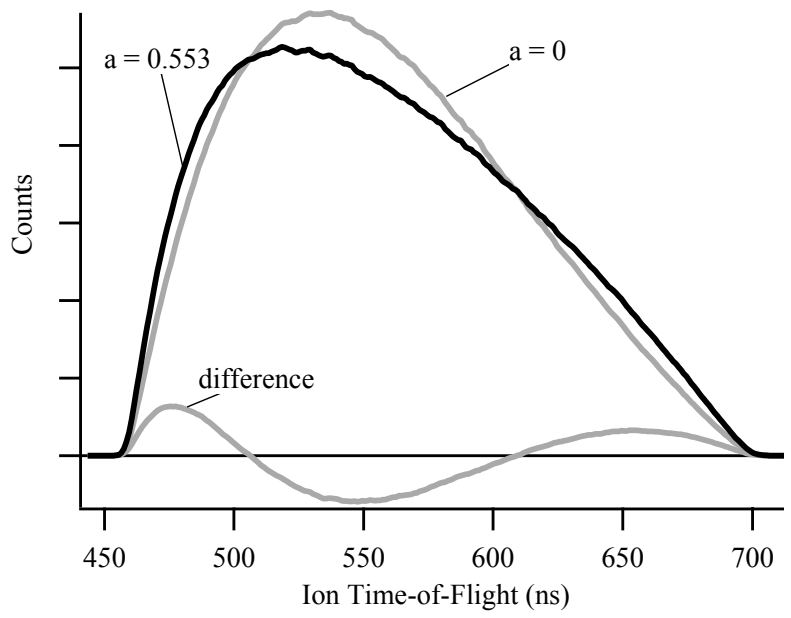

Fig. 1. Monte Carlo simulation of the time-of-flight spectra for ${ }^{21} \mathrm{Ne}^{+}$given $a_{\beta v}=0.553$ (calculated value) and $a_{\beta v}=0$, and the difference between these two spectra.

trapped atom cloud with respect to the detectors is crucial for determining $a_{\beta v}$. These trap parameters were determined from camera images and measured timeof-flight distributions. We measured the TOF of autoionized dimer ions $\left({ }^{23} \mathrm{Na}_{2}^{+}\right)$

\footnotetext{
${ }^{\mathrm{b}}$ Although the charge state distribution for ${ }^{21} \mathrm{Ne}$ from electron capture is different from the positron decay mode, because of the $\mathrm{K}$ shell vacancy.
} 
as a function of trap position. The distance from the MOT to the ion MCP was verified by measuring the TOF of ${ }^{21} \mathrm{Na}_{2}^{+}$, and by using $\beta$-decay coincidence events of neutral recoils, ${ }^{21} \mathrm{Ne}^{0}$. In the latter case, the rising edge of the TOF spectrum depends on the MOT/MCP distance, independent of the electric field.

\section{Molecular sodium}

As the MOT operates, molecular sodium $\left(\mathrm{Na}_{2}\right)$ is generated via photoassociation during collisions between cold trapped atoms. In sodium, the second molecular excited state manifold is autoionizing. This creates a low-energy electron and a $\mathrm{Na}_{2}^{+}$ion with very low momentum. We detect molecular sodium as coincidence events in the MCP pair with a strongly peaked TOF originating from the trap location. The observation of autoionized dimer molecules implies a population of cold, ground state molecular sodium, since both autoionization and ground state molecules are formed via the same pathways through intermediate short-range molecular states. Molecules with a net magnetic moment can be confined in the magnetic trap formed by the MOT's magnetic field gradient, implying a cold, trapped, molecular population. It is difficult to determine the absolute fraction of trapped ${ }^{21} \mathrm{Na}_{2}$ by measuring the rate of ionized dimers. This would require knowing the spontaneous emission rate and autoionization probabilities of the molecular states. We have measured the rate of autoionized ${ }^{23} \mathrm{Na}_{2}$ dimers as a function of the trapped atomic population: this measures the relative molecular population of the MOT as a function of atomic population, since the formation rate of autoionized dimers must be related to the formation rate of cold, ground state, trapped dimers. We find a strong dependence of the dimer ion rate per trapped atom on the population of the MOT, shown in Fig. 2. This scaling would be very different for other laser trapped species. In our previous measurement, Ref. [7], the result for $a_{\beta v}$ depended on the number of atoms held in the MOT, and this dependence was likely caused by counting beta decay events in which the detected recoil nucleus scattered from a molecular partner. For beta decay occuring in ${ }^{21} \mathrm{Na}_{2}$, the recoil nucleus is created near the molecular partner. The scattering potential for ${ }^{21} \mathrm{Ne}$ -

${ }^{21} \mathrm{Na}$ is not known, and it would depend on the charge of the ${ }^{21} \mathrm{Ne}$. Scattering of the nuclear recoil momentum will randomize the momentum direction, and the momentum would be shared with the molecular partner.

\section{Systematic uncertainties}

To address the recoil scattering from molecular partners, data were acquired with a range of trap populations. Figure 2 suggests that there should be a dependence of $a_{\beta v}$ with trap population, as the molecular population fraction per trapped atom 


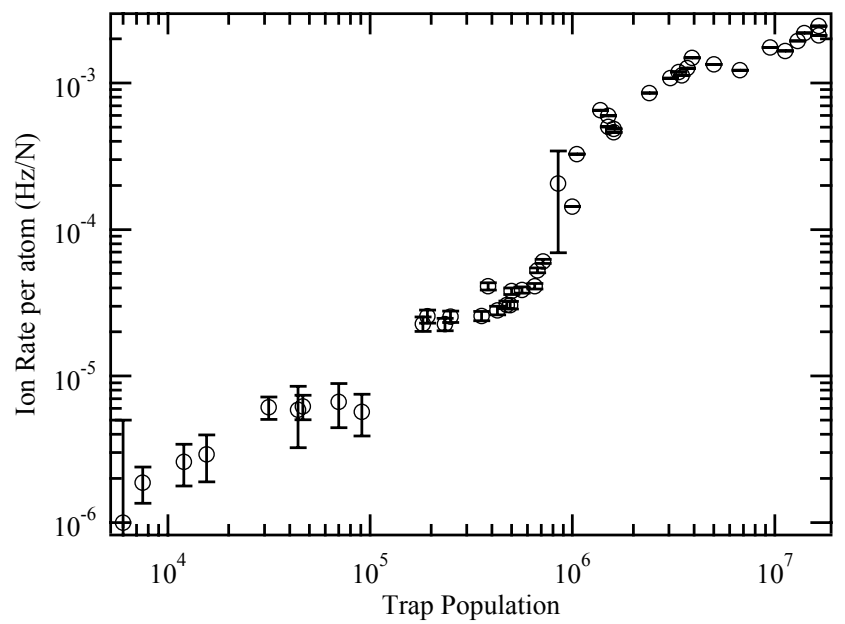

Fig. 2. Rate of detected ${ }^{23} \mathrm{Na}_{2}^{+}$per trapped atom as a function of the trap atomic population.

changes. Data were also acquired using a dark MOT technique, in which the usual trap repumping laser is not incident on the trapped atoms. The average atomic excited state population is greatly reduced, inhibiting the photoassociation process (which requires collisions involving excited state atoms) by nearly three orders of magnitude. In Fig. 3, we show the current data set and the averaged data from [7]. The perturbation on $a_{\beta v}$ from molecular recoil scattering can be estimated by fitting these data to a curve derived from the measured rate of molecular ions (per atom) as a function of trap population, Fig. 2. This fit gives a negligible correction $(0.05 \%)$ to the current data set for an extrapolation to zero trap population, while also supporting the plausibility of extrapolating the data from [7].

A component of the background in the TOF spectrum data is caused by the coincident detection of a $\beta^{+}$or $\gamma$ with the electron MCP and a positively charged recoil ${ }^{21} \mathrm{Ne}$ ion. These events have a biased kinematic distribution of recoil momenta. It is difficult to accurately calculate the contribution to the TOF data from these $\beta^{+}$-triggered events, since the detection efficiency of the electron MCP as a function of $\beta^{+}$energy and number of incident shakeoff electrons is not precisely known. This background subtraction contributes a $0.5 \%$ uncertainty in $a_{\beta v}$. A small correction is necessary to the measured $a_{\beta v}$ because internal conversion of the excited-state $\left(5 / 2^{+}, 350.7 \mathrm{kev}\right)$ causes the excited state contribution in each charge state of ${ }^{21} \mathrm{Ne}$ to deviate from the $\beta$ decay branching ratio. Internal conversion causes in an inner shell vacancy and Auger electron loss, giving higher charge states for excited state decays, or effectively larger decay branching ra- 


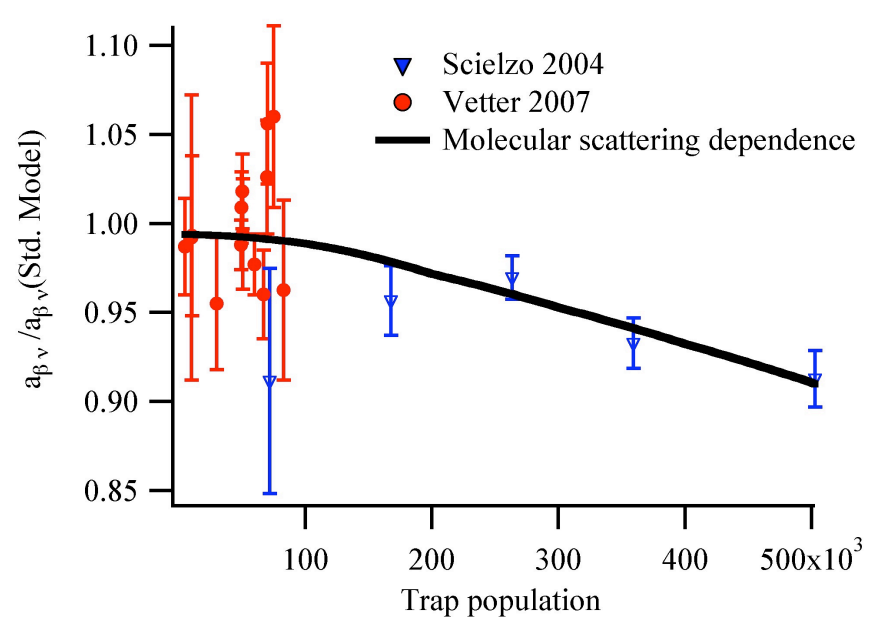

Fig. 3. $a_{\beta v}$ at different trap populations. Data from [7] are shown. The solid line is a fit to the dependence of a perturbation from trapped ${ }^{21} \mathrm{Na}_{2}$.

tio to the higher charge states. Since the $\beta-v$ correlation is measured only for daughter ${ }^{21} \mathrm{Ne}$ that have lost $\geq 2$ electrons, the ionization process could lead to systematic effects. A calculation in [11] indicated that nuclear recoil should increase ionization for the fastest recoils, necessitating a correction of $0.6 \pm 0.3 \%$ to $a_{\beta v}$, and estimates this effect in other candidate $\beta$ decay correlation measurement systems.

\section{Conclusions}

Our result, averaging the data in Fig. 3 and applying several small corrections, is $a_{\beta v}=0.5502(38)(46)$, where the first uncertainty is statistical and the second systematic. This is to be compared with the calculated value $a_{\beta v}=0.553(2)$, which assumes Standard Model couplings, and which has an uncertainty limited by the decay parameters (half-life, $Q$ value, and branching ratios).

In the available high-precision experiments of $a_{\beta v}$, the beta neutrino momentum correlation is measured essentially from the recoil energy spectrum, meaning that the observable is

$$
\tilde{a}=\frac{a}{1+b_{\text {Fierz }} \frac{m_{e}}{\left\langle E_{\beta}\right\rangle}},
$$

with $a$ and $b_{\text {Fierz }}$ as in Eqs. 2 and 4, and $\left\langle E_{\beta}\right\rangle$ the mean energy of the $\beta^{ \pm}$. Combining the results of several precise measurements of the beta-neutrino correlation 
$\tilde{a}$ produces a limit on the existence of non-Standard Model scalar and tensor current couplings, shown in Fig. 4. To generate this exclusion plot, we assume "normal helicity" $C_{S}=C_{S}^{\prime}$ and $C_{T}=C_{T}^{\prime}$, and we assume that $\operatorname{Im}\left(C_{S}\right)=\operatorname{Im}\left(C_{T}\right)=0$ (i.e. time reversal invariance). Each system, by virtue of the different contriubtions of Fermi and Gamow-Teller transition strengths, and different mean beta energies for the Fierz term contribution, yields a different sensitivity to possible scalar and tensor contributions. The one, two, and three $\sigma$ contours are derived from the combined constraints offered by the different measurements of $\tilde{a}$. For comparison, we also show (as a vertical grey bar) the allowed region of scalar coupling constants consistent with the analysis of superallowed Fermi decays $C_{S} / C_{V}=-(0.00005 \pm 0.00130)$ [12]. The allowed region calculated only from measurements of $a_{\beta v}$ is roughly $C_{S} / C_{V}, C_{T} / C_{A}<0.01$, which (naïvely) probes for new couplings at an energy scale roughly $10 M_{W}$.

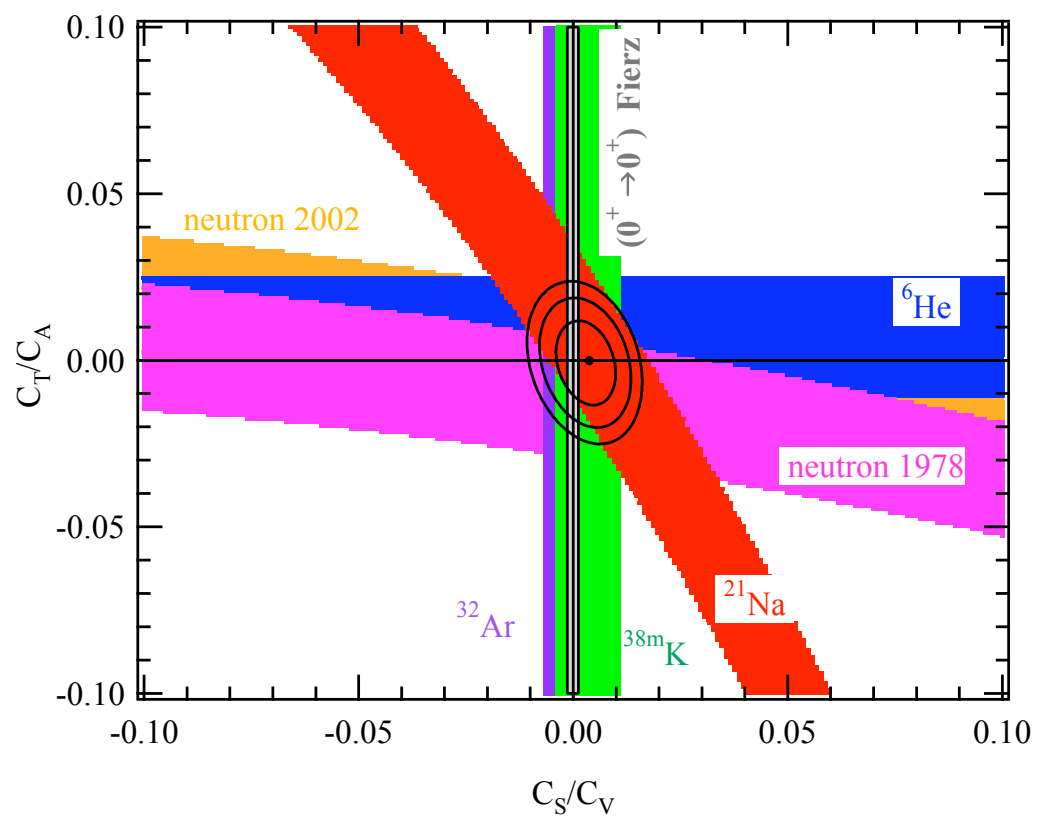

Fig. 4. Amplitudes of calar and tensor coupling constants allowed by precise measurements of $\beta_{3 v}$. The 1,2, and $3 \sigma$ contours for the combined limit are shown. The vertical grey band is allowed by the limit on the Fierz term from superallowed $\left(0^{+} \rightarrow 0^{+}\right)$Fermi decays [12]. The systems are ${ }^{6} \mathrm{He}$ [13] (blue), neutron [14] (pink) and [15] (orange), ${ }^{21} \mathrm{Na}$ (this work, red), ${ }^{38 m} \mathrm{~K}$ [4] (green), and ${ }^{32} \mathrm{Ar}$ [16] (violet). A measurement for ${ }^{23} \mathrm{Ne}$ [17] is not shown, but is used to calculate the allowed region. 


\subsection{Outlook for atom traps}

Measurements of the beta-neutrino correlation coefficient using laer-trapped atoms could likely be improved to the $0.1 \%$ level of precision, which would offer somewhat more potent limits on Beyond Standard Model physics. This would be particularly interesting in the case of ${ }^{6} \mathrm{He}$, which has been discussed as a candidate for experiments in several forums, because of its pure Gamow-Teller transition, which cleanly constrains tensor couplings. The decay of ${ }^{18} \mathrm{Ne}$ would offer an opportunity to measure both pure Fermi and pure Gamow-Teller beta decays in the same nucleus, if the excited state is tagged in the detection. In our experiment, the experiment described in Ref. [4], and the work described in talks at this workshop by Jungmann and by Behr, the achievable uncertainties are limited by some common issues. Careful attention must be paid to detector response calibration, electric field calibrations, and measurements of the trapped atom cloud distribution and location. It will be challenging to reduce the uncertainty from the absolute detection efficiencies of the MCP's for electrons and recoil ions, which relates to background subtraction, rate and position dependent detection efficiency, and more precise inclusion of electron capture events. The neutral recoil atoms offer useful information and require detectors with good efficiency for the lowenergy neutrals. We must also suppress molecular formation in laser traps, and characterize this issue for beta decay and other applications of laser-trapped radioactive species. This is less serious when using isotopes with shorter half-lives, in which a smaller trap population can produce a statistically useful experimental result, while still having a low atomic photoassociation rate. The uncertainty in the momentum dependence of the recoil ionization probability could be reduced by a more sophisticated treatment than given in Ref. [11]. The uncertainty in the predicted value for $a_{\beta v}$ in ${ }^{21} \mathrm{Na}(0.4 \%)$ is dominated by the uncertainties in the half-life and decay branching ratio could also soon be a limiting factor, as in other systems.

This work was supported by the Director, Office of Science, Office of Basic Energy Sciences, U.S. Department of Energy under Contract No. DE-AC0376 SF00098.

\section{References}

1. N. Severijns, M. Beck and O. Naviliat-Cuncic, Rev. Mod. Phys. 78, p. 991 (2006).

2. O. Naviliat-Cuncic, T. A. Girard, J. Deutsch and N. Severijns, J. Phys. G 17, p. 919 (1991).

3. F. Glück, Nucl. Phys. A 628, p. 493 (1998).

4. A. Gorelov et al., Phys. Rev. Lett. 94, p. 142501 (2005).

5. D. Feldbaum, H. Wang, J. Weinstein, D. Vieira and X. Zhao, Phys. Rev. A 76, p. 
051402(R) (2007).

6. H. Wilschut et al., Hyperfine Interactions 174, p. 97 (2007).

7. N. D. Scielzo, S. J. Freedman, B. K. Fujikawa and P. A. Vetter, Phys. Rev. Lett. 93, p. 102501 (2004).

8. N. D. Scielzo, S. J. Freedman, B. K. Fujikawa, I. Kominis, R. Maruyama, P. A. Vetter and J. R. Vieregg, Nucl. Phys. A 746, p. 677c (2004).

9. J. D. Jackson, S. B. Treiman and H. W. Wyld, Phys. Rev. 106, p. 517 (1957).

10. B. R. Holstein, Rev. Mod. Phys. 46, p. 789 (1974).

11. N. D. Scielzo, S. J. Freedman, B. K. Fujikawa and P. A. Vetter, Phys. Rev. A 68, p. 022716 (2003).

12. J. C. Hardy and I. S. Towner, Phys. Rev. C 71, p. 055501 (2005).

13. C. H. Johnson, F. Pleasonton and T. A. Carlson, Phys. Rev. 132, p. 1149 (1963).

14. C. Stratowa, R. Dobrozemsky and P. Weinzierl, Phys. Rev. D 18, p. 3970 (1978).

15. J. Byrne, P. G. Dawber, M. G. D. van der Grinten, C. G. Habeck, F. Shaikh, J. A. Spain, R. D. Scott, C. A. Baker, K. Green and O. Zimmer, J. Phys. G 28, p. 1325 (2002).

16. E. G. Adelberger et al., Phys. Rev. Lett. 83, p. 1299 (1999).

17. T. Carlson, Phys. Rev. 132, p. 2239 (1963). 